\title{
Contribuições e dificuldades da equipe de enfermagem na implementação de cuidados paliativos ao paciente oncológico
}

\author{
Contributions and difficulties of the nursing team in the implementation of palliative care for
} cancer patients

Contribuciones y dificultades del equipo de enfermería en la implementación de cuidados paliativos para pacientes con câncer

Crisley Lorraine Costa Sales ORCID: https://orcid.org/0000-0002-8490-3826 Universidade do Estado de Mato Grosso, Brasi

E-mail: crislorraine012@gmail.com

Joselaine Souto Hall Silva

ORCID: https://orcid.org/0000-0001-5667-8871 Universidade do Estado de Mato Grosso, Brasil E-mail: joselaineshs@unemat.br

Poliana Roma Greve Nodari ORCID: https://orcid.org/0000-0002-6526-4758 Faculdade Estácio do Pantanal (Fapan), Brasil E-mail: poliananodari@ fapan.edu.br

Daiana Alves Vendramel da Costa ORCID: https://orcid.org/0000-0003-2304-1393 Universidade do Estado de Mato Grosso, Brasil E-mail: daivendramel@gmail.com

Thaís Martins dos Santos ORCID: https://orcid.org/0000-0002-4957-9995 Universidade do Estado de Mato Grosso, Brasil E-mail: thais.martins@unemat.br

\begin{abstract}
Resumo
O câncer é uma doença avassaladora, e os cuidados paliativos devem estar presentes a partir do momento em que a doença se torna agressiva e em estágio avançado, sendo uma atribuição da equipe de enfermagem planejá-los e oferecê-los aos pacientes. O objetivo foi analisar as contribuições e dificuldades da enfermagem na implementação dos cuidados paliativos aos pacientes oncológicos terminais. A busca de artigos foi realizada nas bases de dados indexadas na Biblioteca Virtual de Saúde, entre julho e novembro de 2020, utilizando a metodologia Prisma. Foram incluídos na amostra 14 artigos que contemplaram o tema, sendo 92,8\% no idioma inglês e apenas 7,1\% nacional. Além disso, 35,7\% são produções de países europeus e 28,5\% são da América do Norte. O maior número de publicações em países europeus pode estar relacionado ao fato de que a Europa é o berço do estudo sobre os cuidados paliativos. Constatou-se, entre os 14 estudos, a importância da enfermagem oncológica na prestação de cuidados de fim de vida. No entanto, esses profissionais têm dificuldade em lidar com as dores físicas e emocionais dos pacientes, receios em falar sobre antecipação do planejamento dos cuidados paliativos, falta de confiança em comunicar com o paciente em momentos de preocupações, medos e anseios no que diz respeito ao processo morte e morrer, apego emocional, dúvidas de quais são suas atribuições reais no cuidado e limitações como a falta de tempo para realizar formação/capacitação presenciais ou online.
\end{abstract}

Palavras-chave: Câncer; Cuidados de enfermagem; Paciente terminal; Enfermagem oncológica.

\begin{abstract}
Cancer is an overwhelming disease and palliative care must be present from the moment the disease becomes aggressive and at an advanced stage, and it is an assignment of the nursing team to plan and offer it to patients. The objective was to analyze the contributions and difficulties of nursing in the implementation of palliative care to terminal cancer patients. The search for articles was performed in the databases indexed in the Virtual Health Library, between July and November 2020, using the Prisma methodology. The sample included 14 articles that addressed the theme, $92.8 \%$ in the English language and only $7.1 \%$ national. In addition, $35.7 \%$ are productions from European countries and $28.5 \%$ are from North America. The largest number of publications in European countries may be related to the fact that Europe is the birthplace of the study on palliative care. The studies included the importance of
\end{abstract}


oncology nursing in the provision of end-of-life care. However, these professionals have difficulty dealing with the physical and emotional pain stems from patients, fears about anticipating palliative care planning, lack of confidence in communicating with the patient in times of concern, fears and longings regarding the death and dying process, emotional attachment, doubts of what are their real duties in care and limitations such as lack of time to perform faceto-face or online training/training.

Keywords: Cancer; Nursing care; Terminal pacient; Oncology nursing.

\section{Resumen}

El cáncer es una enfermedad abrumadora y los cuidados paliativos deben estar presentes desde el momento en que la enfermedad se vuelve agresiva y en una etapa avanzada, y es una asignación del equipo de enfermería planificar y ofrecerla a los pacientes. El objetivo era analizar las contribuciones y dificultades de la enfermería en la implementación de cuidados paliativos a pacientes con cáncer terminal. La búsqueda de artículos se realizó en las bases de datos indexadas en la Biblioteca Virtual de Salud, entre julio y noviembre de 2020, utilizando la metodología Prisma. La muestra ha incluido 14 artículos que abordaron el tema, 92,8\% en el idioma inglés y sólo 7,1\% nacional. Además, el 35,7\% son producciones de países europeos y el 28,5\% de América del Norte. El mayor número de publicaciones en los países europeos puede estar relacionado con el hecho de que Europa es el lugar de nacimiento del estudio sobre los cuidados paliativos. Los estudios incluyeron la importancia de la enfermería oncológica en la prestación de atención al final de la vida. Sin embargo, estos profesionales tienen dificultades para lidiar con el dolor físico y emocional derivados de los pacientes, los temores de anticipar la planificación de cuidados paliativos, la falta de confianza en la comunicación con el paciente en momentos de preocupación, los temores y anhelos con respecto al proceso de muerte y muerte, el apego emocional, las dudas de cuáles son sus deberes reales en la atención y limitaciones como la falta de tiempo para realizar entrenamiento/entrenamiento cara a cara o en línea.

Palabras clave: Cáncer; Cuidado de enfermera; Paciente terminal; Enfermería oncológica.

\section{Introdução}

O câncer é o principal problema de saúde pública no mundo e já está entre as quatro principais causas de morte prematura (antes dos 70 anos de idade) na maioria dos países (Instituto Nacional de Câncer José Alencar Gomes da Silva [Inca], 2019). A mais recente estimativa mundial, realizada no ano de 2018, aponta que ocorreram no mundo 18 milhões de casos novos de câncer e 9,6 milhões de óbitos, sendo o câncer de pulmão o mais incidente no mundo (2,1 milhões) seguido pelo câncer de mama (2,1 milhões), cólon e reto (1,8 milhão) e próstata (1,3 milhão) (Inca, 2019).

Segundo o relatório da incidência de câncer no Brasil de 2020 elaborado pelo Inca (2019), a estimativa no Brasil para cada ano do triênio 2020-2022 aponta que ocorrerão 625 mil casos novos de câncer (450 mil, excluindo os casos de câncer de pele não melanoma), sendo que o câncer de pele não melanoma será o mais incidente (177 mil), seguido pelos cânceres de mama e próstata (66 mil cada), cólon e reto (41 mil), pulmão (30 mil) e estômago (21 mil).

O início do tratamento de um câncer é exigente e rigoroso, pois a princípio seu objetivo é a cura ou remissão da doença, o que acarreta esperança para o doente e familiar, mas quando se confirma um câncer terminal (sem chance de cura), isso afeta diretamente os envolvidos no tratamento, interferindo nos aspectos psicossociais. Os cuidados paliativos devem estar presentes a partir do momento em que a doença se torna agressiva e em estágio avançado. (Figueiredo et al., 2009).

A Organização Mundial de Saúde (OMS), em 1990, conceituou o termo cuidado paliativo, e em 2002, redefiniu como sendo a assistência prestada aos pacientes e familiares diante de doenças que ameaçam a continuidade da vida com vistas a promover a qualidade de vida através da prevenção e alívio do sofrimento. O cuidado paliativo vai além de procedimentos baseados na terminalidade, é uma atenção especial, e inclui um cuidado sem preceitos e paradigmas, que valorize a singularidade do sujeito e seus processos familiares, desde o diagnóstico até o pós-morte. Desta forma, é necessário que a equipe oncológica paliativa seja composta por diversos profissionais, uma equipe multiprofissional, que irá atuar em todas as dimensões (Silva, 2017).

A enfermagem é parte desta equipe, e cabe a esses profissionais prestarem uma assistência voltada a condutas éticas e humanas visando prevenção e controle de sintomas, autonomia e independência, intervenção espiritual e psicossocial, comunicação seja verbal ou não verbal e assistência ao familiar no pós-morte (Carvalho \& Parsons, 2012). Além dessas 
condutas, a enfermagem oncológica deve desenvolver ações com o paciente e seus familiares, buscando realizar condutas de conforto, esclarecimento, procedimentos, escutas, acolhimento e cuidados básicos e fisiopatológicos. Contudo, esses profissionais acabam tornado educadores em saúde baseados em conceitos claros e objetivos, visando conforto e bem estar para os pacientes (Hermes \& Lamarca, 2013).

A reflexão sobre esse processo pode ser preocupante, pois os profissionais de enfermagem que passam o maior tempo com os pacientes sofrem desgastes emocionais devido ao estado do paciente (doença, sofrimento, dor e morte) e pela rotina de trabalho, o que pode interferir no cuidado se não houver conhecimento suficiente e formação na área (Paiva et al., 2014).

A partir do momento em que o profissional de enfermagem passar por capacitações, formações e adquirirem conhecimentos científicos, sua percepção frente ao paciente terminal se modifica. A compreensão dos múltiplos fatores sobre o adoecimento direciona a outros rumos, e esses novos horizontes proporcionam uma observação e orientação mais detalhada, atuação ampla e diversificada, e o profissional mais preparado. Esses fatores desencadeiam aspectos que visam identificar os pontos positivos e negativos para evolução de cada cuidado (Carmo \& Oliveira, 2015).

Diante do exposto, objetivou-se investigar quais são as contribuições e dificuldades da equipe de enfermagem na prestação de cuidados paliativos aos pacientes oncológicos terminais.

\section{Metodologia}

Trata-se de uma revisão integrativa da literatura de natureza quali-quantitativa, com a finalidade de reunir estudos científicos publicados entre os anos de 2010 a 2020 abordando o objeto investigado de maneira sintetizada, abrangente e ordenada - permitindo buscar, avaliar e resumir evidências disponíveis.

Conforme Soares et al. (2014), a revisão integrativa consiste em um método de reunião e síntese de resultados de investigações, que absorve as preocupações da área com as teorias que fundamentam as práticas do cuidado de enfermagem e, nesse sentido, agrega revisão de teorias à já conhecida e tradicional revisão de estudos empíricos. Para Ercole et al. (2014) este tipo de revisão tem um potencial maior de desempenhar métodos na prática baseadas em evidências, além de proporcionar ao pesquisador elaborar pesquisas relacionadas a conceitos e análises metodológicas e de teorias de assuntos relativos aos cuidados de saúde, relevantes para enfermagem.

Sobre a natureza, Pereira et al. (2018) descrevem que pesquisas de métodos qualitativos podem se transformar em quantitativas quando se utiliza de questões fechadas. Os mesmos autores afirmam que, enquanto o método qualitativo é aquele no qual é importante a interpretação por parte do pesquisador com suas opiniões sobre o fenômeno em estudo, no método quantitativo, faz-se a coleta de dados quantitativos ou numéricos por meio do uso de medições de grandezas, sendo que este enfoque matemático muitas vezes torna-se importante por possibilitar a previsão dos acontecimentos. O método quantitativo é capaz de gerar conjuntos ou massas de dados que podem ser analisados por meio de técnicas matemáticas como é o caso das porcentagens, estatísticas e probabilidades, métodos numéricos, métodos analíticos e geração de equações e/ou fórmulas matemáticas aplicáveis a algum processo (Pereira et al., 2018).

A abordagem metodológica deste estudo consiste nas seis etapas da Pesquisa Baseada em Evidências (PBE) (Mendes et al., 2008): 1) estabelecer a hipótese ou a pergunta da revisão; 2) busca ou amostragem na literatura; 3) categorização dos estudos; 4) análise crítica dos estudos; 5) interpretação dos resultados, e; 6) apresentação da revisão integrativa.

A busca dos estudos primários foi realizada no período de julho a novembro de 2020, por meio das bases de dados eletrônicas em saúde indexadas no portal da Biblioteca Virtual de Saúde (BVS), sendo elas: Literatura Latino-Americana e do Caribe em Ciências da Saúde (Lilacs), Medical Literature Analysis and Retrieval System Online (Medline), Índice Bibliográfico Español en Ciencias de la Salud (Ibecs) e Bases de Dados de Enfermagem (BDENF-Enfermagem). Essa 
abrangência visa garantir publicações de diversas áreas de pesquisa em saúde, permitindo uma busca mais detalhada e refinada em sua execução.

Vale ressaltar que, para o levantamento de estudos na BVS, foram utilizados os descritores em Ciências da Saúde $(D e C S)$ e suas combinações no idioma português, inglês e espanhol, sendo eles: "equipe de enfermagem", câncer, "cuidados paliativos na terminalidade da vida", "paciente terminal" e "enfermagem oncológica". As combinações dos DeCS supracitados foram feitas de forma controlada a partir do operador booleano and, formando as expressões de busca, conforme apresentado na (Tabela 1), juntamente com o total de registros obtidos após a busca na BVS, sem aplicação dos critérios de seleção e elegibilidade.

Tabela 1 - Expressões de busca utilizadas a partir da combinação dos DeCS controlados pelo operador booleano and pelo total de registros obtidos brutos na BVS sem aplicação dos critérios de seleção.

\begin{tabular}{l|c}
\hline Expressões de busca & Total de registros \\
\hline Paciente terminal and enfermagem oncológica & 228 \\
Câncer and cuidados paliativos na terminalidade da vida & 2.118 \\
Equipe de enfermagem and cuidados paliativos na terminalidade da vida & 389 \\
\hline
\end{tabular}

Fonte: Autores (2021).

Para a seleção de artigos, utilizou-se o Prisma (2009) - Instrumento de Análise dos Estudos de Revisão Integrativa que tem como propósito ajudar os autores a melhorarem as pesquisas de revisão e consiste em um checklist com 27 itens. Para este estudo foi realizada uma adaptação com quatro categoriais: 1) Identificação; 2) Seleção; 3) Elegibilidade; 4) Inclusão.

Como critérios de elegibilidade para a obtenção das produções, foram adotados: a) critérios de inclusão: artigos completos; publicados nos últimos dez anos; disponíveis nos idiomas português, espanhol e inglês; b) critérios de exclusão: artigos de revisão; artigos completos que não responderam ao objetivo do estudo; textos duplicados; teses, dissertações e monografias.

A busca e seleção dos artigos foi realizada, criteriosamente, por duas pesquisadoras, para evitar possíveis vieses de seleção. Dos 14 artigos avaliados para a elegibilidade, é importante ressaltar que, nas situações nas quais os resumos não apresentaram informações concisas, procedeu-se a leitura integral do texto para verificar se havia a necessidade de removê-lo da amostra.

Após a seleção dos artigos, as pesquisadoras procederam à tabulação e categorização dos dados através das seguintes variáveis: autores, ano de publicação, base dados indexada, revista/periódico, Qualis Capes, metodologia utilizada (amostra, coleta e análise de dados) e principais resultados relacionados ao objeto de estudo (atribuições do enfermeiro e as dificuldades).

Os dados foram, então, analisados e apresentados de forma quantitativa (\%) através de tabelas, apresentando o perfil dos artigos publicados sobre o tema de estudo, bem como de forma qualitativa, confrontando os principais resultados obtidos com o que se tem disponível na literatura. O processo de coleta de dados até a obtenção dos artigos para a análise, está apresentado na figura 1 a partir da elaboração de um diagrama baseado na metodologia Prisma (2009). 
Figura 1 - Processo de obtenção e seleção dos artigos para a análise, seguindo o instrumento Prisma.

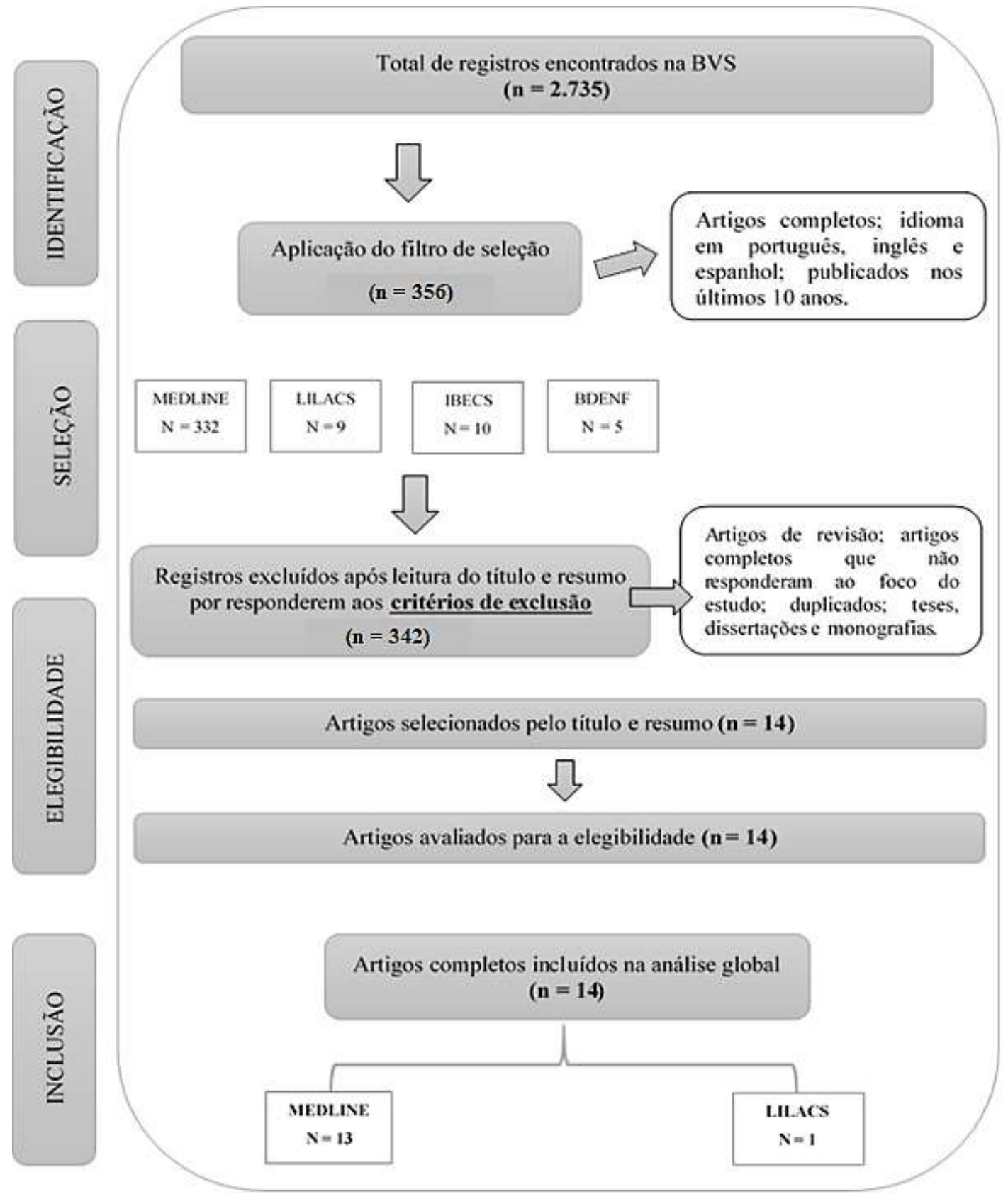

Fonte: Adaptado de Medeiros et al. (2019).

\section{Resultados e Discussão}

A análise inicial do perfil dos 14 artigos evidenciou que 64,3\% das produções foram publicadas nos últimos cinco anos, sendo 35,7\% delas nos anos de 2019 a 2020, o que mostra o interesse recente sobre o tema pesquisado. Em relação ao Qualis Capes, destaca-se que 64,3\% dos periódicos estão classificados atualmente entre os Qualis B2 a A1 (Tabela 2).

Outro destaque são as publicações estrangeiras disponíveis em língua inglesa com 92,8\% das produções, e apenas 7,1\% equivale a publicação nacional. Além disso, 35,7\% são produções de países europeus (Alemanha, Holanda, Inglaterra e Suécia) e 28,5\% são da América do Norte (Canadá e Estados Unidos). Apenas 7,1\% é produção brasileira. 
Tabela 2 - Perfil dos artigos incluídos na análise de acordo com as variáveis autor, ano de publicação, idioma de publicação, base de dados indexada, periódico e Qualis Capes.

\begin{tabular}{|c|c|c|c|c|c|c|}
\hline n. ${ }^{\circ}$ & $\begin{array}{c}\text { Autores/ } \\
\text { Ano de Publicação } \\
\end{array}$ & Idioma & País & $\begin{array}{c}\text { Base de } \\
\text { Dados }\end{array}$ & Periódico & $\begin{array}{l}\text { Qualis } \\
\text { Capes } \\
\end{array}$ \\
\hline A 1 & $\begin{array}{l}\text { Samara et al. } \\
2013\end{array}$ & Inglês & Austrália & Medline & $\begin{array}{c}\text { International Journal Of Evidence- } \\
\text { Based Healthcare }\end{array}$ & B1 \\
\hline A 2 & $\begin{array}{l}\text { Phungrassami et al. } \\
2013\end{array}$ & Inglês & Tailândia & Medline & Journal of Palliative Care & B5 \\
\hline A 3 & $\begin{array}{l}\text { Henoch et al. } \\
2013\end{array}$ & Inglês & Suécia & Medline & $\begin{array}{c}\text { Journal of Pain and Symptom } \\
\text { Management }\end{array}$ & A1 \\
\hline A 4 & $\begin{array}{l}\text { Leclerc et al. } \\
2014\end{array}$ & Inglês & Canadá & Medline & $\begin{array}{c}\text { Journal Of The American Medical } \\
\text { Directors Association }\end{array}$ & A1 \\
\hline A 5 & $\begin{array}{l}\text { Buchanan et al. } \\
2014\end{array}$ & Inglês & Inglaterra & Medline & $\begin{array}{c}\text { International Journal of Palliative } \\
\text { Nursing }\end{array}$ & B3 \\
\hline A 6 & $\begin{array}{l}\text { Phillips et al. } \\
2015\end{array}$ & Inglês & Austrália & Medline & $\begin{array}{c}\text { Collegian journal } \\
\text { Elsevier }\end{array}$ & $\mathrm{C}$ \\
\hline A 7 & $\begin{array}{l}\text { Veldhuise et al. } \\
2016\end{array}$ & Inglês & Holanda & Medline & $\begin{array}{c}\text { International Journal of Palliative } \\
\text { Nursing }\end{array}$ & B3 \\
\hline A 8 & $\begin{array}{l}\text { Seow et al. } \\
2016\end{array}$ & Inglês & Canadá & Medline & Journal Of Palliative Medicine & A1 \\
\hline A 9 & $\begin{array}{c}\text { Sugimura et al. } \\
2017 \\
\end{array}$ & Inglês & Japão & Medline & $\begin{array}{c}\text { International Journal of Palliative } \\
\text { Nursing }\end{array}$ & B3 \\
\hline A 10 & $\begin{array}{l}\text { Fliedne et al. } \\
\quad 2019\end{array}$ & Inglês & Alemanha & Medline & Palliative Medicine & $\mathrm{A} 2$ \\
\hline A 11 & $\begin{array}{l}\text { Reiser et al. } \\
2019\end{array}$ & Inglês & $\begin{array}{l}\text { Estados } \\
\text { Unidos }\end{array}$ & Medline & $\begin{array}{l}\text { American Journal Of Hospice And } \\
\text { Palliative Medicine }\end{array}$ & B1 \\
\hline A 12 & $\begin{array}{l}\text { Feldenzer et al. } \\
2019\end{array}$ & Inglês & $\begin{array}{l}\text { Estados } \\
\text { Unidos }\end{array}$ & Medline & $\begin{array}{c}\text { International Journal of Palliative } \\
\text { Nursing }\end{array}$ & B2 \\
\hline A 13 & $\begin{array}{c}\text { Slev et al. } \\
2020 \\
\end{array}$ & Inglês & Holanda & Medline & $\begin{array}{c}\text { European Journal of Oncology } \\
\text { Nursing }\end{array}$ & A1 \\
\hline A 14 & $\begin{array}{l}\text { Schneider et al. } \\
2020\end{array}$ & $\begin{array}{l}\text { Portugu } \\
\text { ês }\end{array}$ & Brasil & $\begin{array}{c}\text { Lilacs } \\
\text { Express }\end{array}$ & Cienc Cuid Saude & B2 \\
\hline
\end{tabular}

Fonte: Autores (2021).

O maior número de publicações em países europeus pode estar coincidentemente relacionado ao fato de que a Europa é o berço do estudo sobre os cuidados paliativos. De acordo com Gomes e Othero (2016), os cuidados paliativos surgiram oficialmente como prática distinta na área da atenção em saúde nos anos 60 , no Reino Unido, tendo como pioneira a médica Cicely Saunders, que também era assistente social e enfermeira, e iniciou o movimento dos cuidados paliativos que incluía a assistência, o ensino e a pesquisa.

A partir disso, o movimento foi trazido para a América do Norte pela psiquiatra Elisabeth Kübler-Ross, que teve contato com os trabalhos de Saunders. No entanto, foi apenas em 1990, que OMS reconheceu e recomendou os cuidados paliativos, pela primeira vez para 90 países e em 15 idiomas o seu conceito e princípios, sendo que, tal definição foi inicialmente voltada para os portadores de câncer, visando os cuidados de final de vida (Gomes \& Othero, 2016). Além disso, antes mesmo de ser reconhecido e recomendado pela OMS, estudiosos e pesquisadores europeus fundaram no ano de 1988 a European Association for Palliative Care em Milão, Itália.

Em contraponto à Europa, no Brasil, que no presente estudo apresentou apenas uma publicação, a criação de instituições voltadas aos cuidados paliativos aconteceu somente a partir de 1996 com a fundação do Instituto Nacional do Câncer (Inca), hospital oncológico do Ministério da Saúde, que atualmente possui o serviço mais completo em cuidados paliativos no país. Foi então, no ano de 1997, criada a Associação Brasileira de Cuidados Paliativos, com o importante papel de formação de profissionais na área (Pastrana et al., 2012). 
Levanta-se aqui, diante dos fatos apresentados, que a Europa por ser precursora dos cuidados paliativos e, por ter tido a primeira instituição voltada para tal área da saúde, produz e divulga mais produções e conhecimento científico acerca do tema. Ademais, sabendo que os cuidados paliativos visam o cuidado no final da vida, isto também pode estar atrelado ao alto índice de envelhecimento nos países europeus, isto porque, a Itália é o segundo país com mais idosos no mundo, ficando atrás somente do Japão.

De encontro à essa análise, o Inca (2019) traz que a incidência e a mortalidade por câncer vêm aumentando no mundo, em parte pelo envelhecimento, pelo crescimento populacional, como também pela mudança na distribuição e na prevalência dos fatores de risco de câncer, especialmente aos associados ao desenvolvimento socioeconômico, uma vez que, nos países com maior Índice de Desenvolvimento Humano (IDH), as taxas de incidência foram de duas a três vezes maiores que as dos países de médio ou baixo IDH.

Referente à análise sobre as contribuições e dificuldades da enfermagem na implementação dos cuidados paliativos, constatou-se, entre os 14 estudos, a importância da enfermagem oncológica na prestação de cuidados de fim de vida. No entanto, foi notório o quanto esses profissionais têm dificuldade em lidar com as dores físicas e emocionais dos pacientes, receios em falar sobre antecipação do planejamento dos cuidados paliativos, falta de confiança em comunicar com o paciente em momentos de preocupações, medos e anseios no que diz respeito ao processo de morte e morrer, apego emocional, dúvidas de quais são suas atribuições reais no cuidado e limitações como a falta de tempo para realizar formação/capacitação presenciais ou on-line e discutir com o paciente sobre o momento vivenciado.

De acordo com Fernandes et al. (2013), os cuidados paliativos englobam o processo de morte e morrer e o luto é uma das áreas importantes de intervenção. Os autores destacam que é nesse processo que o paciente e a família têm a possibilidade de compreender que a morte é real e a partir dessa experiência, inicia-se uma nova visão sobre o mundo, estabelecimento de novas concepções, a vivência do luto e o planejamento dos momentos que lhe restam. É neste processo que a equipe de enfermagem oncológica terminal irá contemplar essa nova fase do paciente e da família.

Os estudos em análise revelaram também a importância dos cuidados primários, o saber trabalhar e assistir o paciente desde o seu diagnóstico para não haver interferências significativas na relação paciente e profissional de enfermagem, e na não adesão do paciente ao tratamento, a negação de não se comunicar sobre o que se passa, o que na maioria das vezes ocasiona entraves.

Samara et al. (2013) avaliaram a aceitação de enfermeiros e médicos da área da oncologia sobre o Planejamento de Cuidados Avançados (ACP) a partir de uma auditoria pré e pós implementação do ACP. Esta ferramenta permite aos pacientes a oportunidade de discutir e planejar o fim da vida e de aliviar problemas futuros. Identificaram que as maiores limitações observadas foi a falta de tempo para a realização de formações/capacitações, a resistência dos pacientes em relação a antecipação do planejamento dos cuidados e ainda, enfermeiros relatando que é responsabilidade do médico intervir com reuniões e conversas com os pacientes sobre ACP. Os autores refletiram sobre o quanto ainda os enfermeiros precisam aprender a construir a confiança do paciente e trabalhar ao máximo a importância do planejamento nos cuidados paliativos.

Reforçando a necessidade de maior autonomia e liderança na tomada de decisões pela enfermagem, Veldhuise et al. (2016), em seu estudo para avaliar as práticas de enfermagem para a avaliação da ansiedade no paciente com câncer, identificaram que estratégias multifacetadas, liderança e educação de enfermeiras na avaliação e análise da ansiedade são necessárias para melhorar o gerenciamento de sintomas em pacientes com câncer avançado.

Phungrassami et al. (2013) descreveram um modelo de cuidados paliativos hospitalares, no qual foi utilizado um banco de dados de hospitais registrado com as políticas e estratégias do Ministério da Saúde Pública da Tailândia. Neste estudo, foram incluídos profissionais enfermeiros, médicos e outros da saúde com tempo de trabalho em cuidados paliativos. Constatou-se que na maioria dos hospitais 59\% dos profissionais possuíam, no mínimo, uma semana de treinamento, 
conferências, capacitações em cuidados paliativos. Entretanto, os autores revelam que a Tailândia está apenas começando a educação em cuidados paliativos, pois ainda existem muitos hospitais que os profissionais não são treinados para contribuir com um cuidado paliativo de alta qualidade e de forma mais humanizada.

No estudo de Phillips et al. (2015), os autores objetivaram identificar as barreiras e os facilitadores para a avaliação e o manejo da dor do câncer em adultos percebidos por profissionais de saúde australianos. Corroborando com o estudo citado anteriormente, esses autores também identificaram barreiras ao prestar assistência ao paciente oncológico, porém com ênfase na dor do paciente. O estudo apontou dificuldades para o tratamento eficaz da dor oncológica devido à escassez de acesso a intervenções não farmacológicas (89\%), à falta de coordenação por vários provedores (89\%) e ao impacto da distância na capacidade de acessar serviços relacionados à dor para pacientes $(86 \%)$. Pode-se notar que, diferentemente do estudo de Phungrassami et al. (2013), no qual as barreiras que impendiam um atendimento de alta qualidade eram fatores direcionados a falta de formação, conhecimento e receios profissionais, no estudo de Phillips et al. (2015), o fator limitante para o melhor tratamento disponível no ambiente comunitário para pessoas com dor oncológica é o sistema de saúde.

Henoch et al. (2013) descreveram sobre o conhecimento e reflexão dos profissionais da saúde, principalmente da equipe de enfermagem sobre sua utilidade nos cuidados paliativos. Os autores identificaram que muitos profissionais mesmo, com anos de profissão e idade mais avançada, possuem anseios e medos ao falar com os pacientes sobre o fim da vida e principalmente sobre o processo de morte e morrer, e defendiam que não era papel deles discutir e ouvir as preocupações dos pacientes. Após a realização de treinamento com esses profissionais, o estudo destacou que os profissionais que foram treinados para lidar com esses pacientes no final de vida demonstraram mais confiança na comunicação.

A comunicação é um ato imprescindível para as condutas a serem tomadas. A comunicação, a experiência, a formação, conquista da confiança do paciente, intervenções e condições adequadas para se trabalhar são vertentes extremamente necessárias para que os profissionais de enfermagem possam contribuir para o tratamento do paciente oncológico.

Nesse contexto, Feldenzer et al. (2019) tiveram como objetivo compreender os impactos pessoais e profissionais do treinamento oferecido em cuidados paliativos primários pelo Care Management by Oncology Nurses (CONNECT). Os enfermeiros relataram uma sensação de realização pessoal e profissional ao fornecer os cuidados paliativos primários, enquanto observaram também o risco de aumento do apego emocional aos pacientes. Foi evidente que o treinamento melhorou as habilidades de comunicação da enfermagem, e o apoio no local de trabalho ajudou a minimizar o estresse relacionado à incorporação do cuidado paliativo.

Reiser et al. (2019) estudaram os efeitos de um projeto de melhoria da qualidade de vida no Programa de assistência a mulheres com câncer de mama metastático estágio IV. As pacientes tinham entre 50 e 60 anos de idade e estavam há três anos no programa. O projeto tinha como finalidade encaminhar as mulheres para a assistência social e tratamento com cuidados paliativos, conduzido por enfermeiras. Os resultados foram muito positivos após a implementação do projeto. Houve a redução significativa dos problemas de transtorno de ansiedade generalizada e sofrimento, bem como o aumento do bem estar geral.

Buchanan et al. (2014) afirmaram que os enfermeiros têm um papel vital no diagnóstico, avaliação e gerenciamento da dor no câncer avançado. Os autores consideram que as diretrizes da European Oncology Nursing Society (EONS) e o guia de bolso associado são recursos inestimáveis para a enfermagem, e a adoção mais ampla dos princípios descritos nas diretrizes deve levar a uma melhoria no cuidado dos pacientes com esse estado de dor frequentemente difícil de tratar. Desvelando também sobre o papel dos enfermeiros nos cuidados paliativos, Sugimura et al. (2017) afirmaram que essa prática é importante para apoiar também pacientes com dispneia, e os enfermeiros devem possuir o conhecimento e as habilidades necessárias para prestar esse cuidado de forma adequada. 
Schneider et al. (2020) revelaram em seu estudo, em relação ao preparo da equipe para o cuidado à criança em cuidados paliativos, o despreparo em geral. Os profissionais de enfermagem manifestaram diversos sentimentos e percepções: empatia, compaixão, amor, doação, envolvimento, gratificação, impotência, mal-estar e desconforto diante do cuidar de uma criança em cuidados paliativos. Os profissionais descrevem a vontade de manter o paciente vivo. Essas dificuldades relacionam-se diretamente à perspectiva de morte da criança, principalmente daqueles pacientes com os quais o profissional se envolveu mais.

Schneider et al. (2020) afirmam que é necessário encontrar mecanismos de enfrentamento que possibilitem a manutenção de serenidade para assistir bem a criança e sua família nos momentos finais. Para isso, a filosofia de Cuidados Paliativos precisa ser ampliada para uma rede integrada de atenção e respeito às decisões para o final da vida, numa perspectiva interdisciplinar. Os mesmos autores enfatizam sobre a necessidade de lançar um olhar apurado aos profissionais de enfermagem, de modo a favorecer seu empoderamento para o exercício do cuidado ético e sensível que esses pacientes requerem nesse momento existencial.

Como limitação para a realização do presente estudo, destaca-se a obtenção de materiais disponíveis, em grande parte na língua inglesa. A realização da tradução pode ter dificultado a compreensão e interpretação do que os autores encontraram em seus estudos.

\section{Considerações Finais}

A maioria dos profissionais de enfermagem tem dificuldades em aceitar e compreender o processo da terminalidade dos pacientes, uma vez que não são preparados durante a formação acadêmica, o que dificulta o enfrentamento de situações mais complexas da fase final da vida dos pacientes.

Os autores dos artigos selecionados concordam que a equipe de enfermagem é elemento essencial do cuidado ao paciente e família sob os cuidados paliativos a comunicação e a proximidade de vínculo. No entanto, devido aos problemas emocionais provocados pela condição de terminalidade, os enfermeiros se sentem confusos e angustiados, enfrentando situações conflituosas, o que requer um preparo diferenciado desses profissionais.

Portanto, é consenso entre os autores que os profissionais de enfermagem devem receber capacitação, treinamentos e atualizações sobre a temática de cuidados paliativos, bem como, que sejam preferencialmente profissionais especializados na área. É evidente que o conhecimento técnico científico melhora a atuação profissional, uma vez que cabe ao enfermeiro decidir questões importantes e assumir responsabilidades de forma integral no cuidado ao paciente, visando ao bem-estar, alívio da dor, enfrentamento da doença, compreensão de seus desejos, da escuta ativa e da promoção ao conforto.

Como recomendações para estudos futuros, sugere-se a realização de mais produções nacionais, tendo em vista os altos índices de câncer e envelhecimento registrados no Brasil. Recomenda-se a realização de estudos que tragam, de forma mais ampla, o planejamento dos cuidados paliativos na assistência de enfermagem aos pacientes terminais, além de pesquisas de campo que abordem como pode ser realizado o enfrentamento das dificuldades na implementação dos cuidados paliativos não somente pela equipe de enfermagem, mas por toda a equipe multiprofissional que presta assistência a estes pacientes.

\section{Referências}

Buchanan, A., Davies, A., \& Geerling, J. (2014). Breakthrough cancer pain: the role of the nurse. International journal of palliative nursing, 20(3), 126-129. https://doi.org/10.12968/ijpn.2014.20.3.126

Carvalho, R. T. D., \& Parsons, H. A. (2012). Manual de cuidados paliativos ANCP. Porto Alegre; Sulina; (2a ed.) 590 p. ilus, tab. http://biblioteca.cofen.gov.br/wp-content/uploads/2017/05/Manual-de-cuidados-paliativos-ANCP.pdf

Carmo, S. A., \& dos Santos Oliveira, I. C. (2015). Criança com câncer em processo de morrer e sua família: enfrentamento da equipe de enfermagem. Revista Brasileira de Cancerologia, 61(2), 131-138. https://doi.org/10.32635/2176-9745.RBC.2015v61n2.300 
Ercole, F. F., Melo, L. S. D., \& Alcoforado, C. L. G. C. (2014). Revisão integrativa versus revisão sistemática. Revista Mineira de Enfermagem, 18(1), 9-12. http://www.dx.doi.org/10.5935/1415-2762.20140001

Feldenzer, K., Rosenzweig, M., Soodalter, J. A., \& Schenker, Y. (2019). Nurses' perspectives on the personal and professional impact of providing nurse-led primary palliative care in outpatient oncology settings. International journal of palliative nursing, 25(1), 30-37. https://doi.org/10.12968/ijpn.2019.25.1.30

Fernandes, M. A., Evangelista, C. B., Platel, I. C. D. S., Agra, G., Lopes, M. D. S., \& Rodrigues, F. D. A. (2013). Percepção dos enfermeiros sobre o significado dos cuidados paliativos em pacientes com câncer terminal. Ciência \& Saúde Coletiva, 18(9), 2589-2596. http://dx.doi.org/10.1590/S141381232013000900013

Figueiredo, N. M. A., Leite, J. L., Machado, W. C. A., Moreira, M. C., \& Tonini, T. (2009). Enfermagem Oncológica: Conceitos e Práticas Yendis Editora.

Fliedner, M., Zambrano, S., Schols, J. M., Bakitas, M., Lohrmann, C., Halfens, R. J., \& Eychmüller, S. (2019). An early palliative care intervention can be confronting but reassuring: A qualitative study on the experiences of patients with advanced cancer. Palliative medicine, 33(7), 783-792. https://doi.org/10.1177/0269216319847884

Galvão, T, F., Pansani, T. S. A., \& Harrad, D. (2015). Principais itens para relatar revisões sistemáticas e Meta-análises: A recomendação PRISMA*. Epidemiol. Serv. Saúde, Brasília. 10.5123/S1679-49742015000200017.

Gomes, A. L. Z. \& Othero, M. B. (2016). Cuidados paliativos. Estudos Avançados, 30 (88), 155-166. https://dx.doi.org/10.1590/s0103-40142016.30880011

Henoch, I., Danielson, E., Strang, S., Browall, M., \& Melin-Johansson, C. (2013). Training intervention for health care staff in the provision of existential support to patients with cancer: a randomized, controlled study. Journal of pain and symptom management,46(6), 785-794. https://doi.org/10.1016/j.jpainsymman.2013.01.013

Hermes, H. R., \& Lamarca, I. C. A. (2013) Cuidados paliativos: uma abordagem a partir das categorias profissionais de saúde. Ciência e Saúde Coletiva, 18(9), 5577-2588. https://www.scielosp.org/pdf/csc/2013.v18n9/2577-2588/pt

Instituto Nacional de Câncer José Alencar Gomes da Silva [Inca]. (2019). Estimativa 2020: incidência de câncer no Brasil. Inca, 120 p.: il. color. https://www.inca.gov.br/publicacoes/livros/estimativa-2020-incidencia-de-cancer-no-brasil

Leclerc, B. S., Lessard, S., Bechennec, C., Le Gal, E., Benoit, S., \& Bellerose, L. (2014). Attitudes toward death, dying, end-of-life palliative care, and interdisciplinary practice in long term care workers. Journal of the American Medical Directors Association, 15(3), 207-213. https://doi.org/10.1016/j.jamda.2013.11.017

Medeiros, M. O. S. F., Meira, M. V., Fraga, F. M.. R., Sobrinho, C. L. N., Rosa. D. O.S., \& Silva, R. S. (2020). Conflitos bioéticos nos cuidados de fim de vida. Revista Bioética. https://doi.org/10.1590/1983-80422020281375

Mendes, K. D. S., Silveira, R. C. C. P., \& Galvão, C. M. (2008). Revisão integrativa: Método de pesquisa para a incorporação de evidências na saúde e na enfermagem. Revista Texto \& Contexto Enfermagem. https://doi.org/10.1590/S0104-07072008000400018

Paiva, F. C. L., Júnior, J. J. A., \& Damásio, A. C. (2014). Ética em cuidados paliativos: concepções sobre o fim da vida. Rev. Bioét. 22(3), 550560. https://doi.org/10.1590/1983-80422014223038.

Pastrana, T. L. L., Wenk, R. E. J., Monti, C. J., Centeno, C. (2012). Atlas de Cuidados Paliativos de Latinoamérica. ALCP. 1a edición. Houston: IAHPC Press. https://dadun.unav.edu/bitstream/10171/34465/1/Atlas\%20de\%20Cuidados\%20Paliativos\%20de\%20Latinoamerica.pdf

Pereira, A. S., Shitsuka, D. M., Parreira, F. J., \& Shitsuka, R. (2018). Metodologia da pesquisa científica. UFSM. https://repositorio.ufsm.br/bitstream/handle/1/15824/Lic_Computacao_Metodologia-Pesquisa-Cientifica.pdf?sequence=1

Phillips, J. L., Lovell, M., Luckett, T., Agar, M., Green, A., \& Davidson, P. (2015). Australian survey of current practice and guideline use in adult cancer pain assessment and management: The community nurse perspective. Collegian, 22(1), 33-41. https://doi.org/10.1016/j.colegn.2013.11.002

Phungrassami, T., Thongkhamcharoen, R., \& Atthakul, N. (2013). Palliative care personnel and services: a national survey in Thailand 2012. Journal of palliative care, 29(3), 133-139. https://doi.org/10.1177/082585971302900301

Reiser, V., Rosenzweig, M., Welsh, A., Ren, D., \& Usher, B. (2019). The support, education, and advocacy (sea) program of care for women with metastatic breast cancer: a nurse-led palliative care demonstration program. American Journal of Hospice and Palliative Medicine®, 36(10), 864-870. https://doi.org/10.1177/1049909119839696

Rodrigues, A. J., Bushatsky, M., \& Viaro, W. D. (2015). Cuidados paliativos em crianças com câncer: revisão integrativa. Rev enferm UFPE online, Recife, 9(2):718-30. 10.5205/r euol.7028-60723-1-SM.0902201530.

Samara, J., Larkin, D., Chan, C. W., \& Lopez, V. (2013). Advance care planning in the oncology settings. International Journal of Evidence-Based Healthcare, 11(2), 110-114. https://doi.org/10.1111/1744-1609.12011

Schneider, A. S., Ludwig, M. C. F., Neis, M., Ferreira, A. M., \& Issi, H. B. (2020). Percepções e vivências da equipe de enfermagem frente ao paciente pediátrico em cuidados paliativos. Ciênc. cuid. saúde, 9-9. https://doi.org/10.4025/cienccuidsaude.v19i0.41789

Seow, H., Sutradhar, R., McGrail, K., Fassbender, K., Pataky, R., Lawson, B., \& Barbera, L. (2016). End-of-life cancer care: temporal association between homecare nursing and hospitalizations. Journal of palliative medicine, 19(3), 263-270. https://doi.org/10.1089/jpm.2015.0229

Silva, S. M. A. (2017). Os Cuidados ao Fim da Vida no Contexto dos Cuidados Paliativos. Revista Brasileira de Cancerologia. https://doi.org/10.32635/21769745.RBC.2016v62n3.338 
Research, Society and Development, v. 10, n. 3, e30410312460, 2021

(CC BY 4.0) | ISSN 2525-3409 | DOI: http://dx.doi.org/10.33448/rsd-v10i3.12460

Slev, V. N., Molenkamp, C. M., Eeltink, C. M., Pasman, H. R. W., Verdonck-de Leeuw, I. M., Francke, A. L., \& van Uden-Kraan, C. F. (2020). A nurse-led self-management support intervention for patients and informal caregivers facing incurable cancer: A feasibility study from the perspective of nurses. European Journal of Oncology Nursing, 45, 101716. https://doi.org/10.1016/j.ejon.2019.101716

Soares, Cassia Baldini, Hoga, Luiza Akiko Komura, Peduzzi, Marina, Sangaleti, Carine, Yonekura, Tatiana, \& Silva, Deborah Rachel Audebert Delage. (2014). Revisão integrativa: conceitos e métodos utilizados na enfermagem. Revista da Escola de Enfermagem da USP,48(2), 335-345. https://doi.org/10.1590/S0080-6234201400002000020

Sugimura, A., Ando, S., \& Tamakoshi, K. (2017). Palliative care and nursing support for patients experiencing dyspnoea. International journal of palliative nursing, 23(7), 342-351. https://doi.org/10.12968/ijpn.2017.23.7.342

Veldhuisen, H., Zweers, D., de Graaf, E., \& Teunissen, S. (2016). Assessment of anxiety in advanced cancer patients: a mixed methods study. International journal of palliative nursing, 22(7), 341-350. https://doi.org/10.12968/ijpn.2016.22.7.341

World Health Organization (WHO). (2002). National cancer control programmes: policies and managerial guidelines. (2a ed.), WHO. 